\title{
Friction and wear behaviour of 18 polymers in contact with steel in environments of air and water*
}

\author{
J. W. M. Mens \\ Institute of Production and Logistics Research TNO, Apeldoorn (The Netherlands)
}

A. W. J. de Gee

University of Technology, Delf, and Twente University, Enschede (The Netherlands)

(Received March 23, 1991)

\begin{abstract}
18 polymers were characterized with respect to their friction and wear behaviour in contact with steel in environments of air and water. These were six unfilled materials, i.e. polyamide 66 (PA 66), polyoxymethylene (POM), polyethyleneterephthalate (PETP), polyetheretherketone (PEEK), polyphenylenesulphide (PPS) and polyetherimide; also, the same base materials filled with polytetrafluoroethylene (PTFE) or PTFE plus glass fibre were used.

Two types of experiments were performed, i.e. measurement of the coefficient of friction $f$ as a function of the contact temperature $T_{c}$ (" $f-T_{c}$ diagrams"), and measurements of friction and wear in $20 \mathrm{~h}$ tests. The $f-T_{\mathrm{c}}$ diagrams were obtained in air; the $20 \mathrm{~h}$ tests were performed in air and in water at a temperature of $20^{\circ} \mathrm{C}$. In air the polymers ran against rings of ball-bearing steel (AISI 52100); in water the rings were made of stainless steel (AISI 316).

It turned out that in air addition of PTFE was generally beneficial; PA 66, POM, PETP and PEEK, all filled with PTFE, performed exceptionally well (specific wear rates $k$ appreciably lower than $1 \times 10^{-15} \mathrm{~m}^{3} \mathrm{~N}^{-1} \mathrm{~m}^{-1}$ ). In water, however, PTFE did not function at all. Now PA (with or without PTFE), unfilled POM and PETP filled with PTFE and glass fibre met the $k<1 \times 10^{-15} \mathrm{~m}^{3} \mathrm{~N}^{-1} \mathrm{~m}^{-1}$ criterion. With a few exceptions, addition of glass fibre produced unfavourable effects, in air as well as in water.

The observed phenomena are explained tentatively in terms of polymer structures and transfer and wear mechanisms, whereby special attention is paid to the ratio of friction over strength.
\end{abstract}

\section{Introduction}

Since in the early 1970 s Lancaster [1] set an example by writing a comprehensive review paper, surveys on the tribological performance of filled polymers have appeared regularly over the years; see for instance work by Lancaster [2], Tanaka [3], Briscoe [4], Evans and Senior [5], Santner and Czichos [6] and Fusaro [7].

However, a practical need exists for systematic comparisons of polymers with alledgedly favourable tribological properties. Thus Organisatie voor Toegepast Natuurwetenschappelijk Onderzoek TNO; (Organization for Applied Scientific Research

* Paper presented at the International Conference on Wear of Materials, Orlando, FL, U.S.A., April 7-11, 1991. 
in the Netherlands) performed a "multiclient" project on the friction and wear behaviour of polymers which were supposed to perform well at elevated temperatures, whereby particular emphasis was given to the effect of polytetrafluorethylene (PTFE) as a filler.

As usual in this type of research, the work started with the characterization of commercially available materials. In a second phase two of the companies involved (see the Acknowledgments) produced different polymers, in the unfilled condition as well as filled with PTFE, glass fibre or carbon fibre.

In this article some of the results that were obtained are presented and discussed. These results relate to 18 materials, composed on the basis of the following six polymers: polyamide 66 (PA 66); polyoxymethylene (POM); polyethyleneterephthalate (PETP); polyetheretherketone (PEEK); polyphenylenesulphide (PPS); polyetherimide (PEI). The following fillers were also used: $15 \%$ PTFE and 15\% PTFE plus $20 \%$ glass fibre. Experiments were run in dry air with rings of ball-bearing steel (AISI 52100) with a hardness of $8 \mathrm{GPa}(800 \mathrm{HV})$ as mating components and in water at a temperature of $21{ }^{\circ} \mathrm{C}$ with rings of stainless steel (AISI 316) with a hardness of $1.9 \mathrm{GPa}(190 \mathrm{HV}$ ) as mating components.

\section{Types of experiments}

Two types of experiments were performed. Firstly the coefficient of friction $f$ was measured as a function of contact temperature $T_{c}$ (" $f-T_{\mathrm{c}}$ curves"); secondly, friction and wear were measured in $20 \mathrm{~h}$ tests at an environmental temperature $T$ of $20^{\circ} \mathrm{C}$ and a contact temperature $T_{c}$ (" $20 \mathrm{~h}$ tests"). In the latter type of test, $T_{\mathrm{c}}$ varied with the amount of heat produced in the friction interface.

In both cases the TNO tribometer was used.

\subsection{The TNO tribometer}

The TNO tribometer has been described on several previous occasions; see for example Honselaar and de Gee [8]. Figure 1 shows a schematic presentation.

In the present case rectangular $(10 \mathrm{~mm} \times 10 \mathrm{~mm} \times 10 \mathrm{~mm})$ blocks of the polymers to be characterized ( $A$ in Fig. 1) were pressed under a known normal force $F_{N}$ against the curved surface of rotating steel rings (B). During the tests friction between block and ring could be measured with a torque shaft provided with strain gauges. The temperature $T$ was measured with a sliding thermocouple. This was spring loaded with a force of $2 \mathrm{~N}$ against the curved surface of the ring, just outside the friction track in the direct vicinity of the polymer block. The specimens were completely surrounded by an air-tight casing, into which clean and dry air or water could be introduced.

\subsection{Measurement of $f-T_{\mathrm{c}}$ curves}

The measurement of $f-T_{c}$ curves has been described extensively previously by Honselaar and de Gee [8,9]. Briefly, it is carried out as follows.

At specific, freely chosen values of the contact pressure $p$ (or normal force $F_{\mathrm{N}}$ ) and sliding speed $v$ the contact temperature $T_{\mathrm{c}}$ reaches an equilibrium value $T_{\mathrm{c}, \mathrm{e}}$, usually in less than $5 \mathrm{~min}$. When $T_{c}$ has attained equilibrium, it is increased further by computer-controlled inductive heating of the steel ring, the coefficient of friction $f$ being measured all the time. Thus, from $T_{\mathrm{c}}=T_{\mathrm{c}, \mathrm{c}}$ on, $T_{\mathrm{c}}$ becomes an independent variable. Additional variables are the start temperature $T_{c, \min }\left(T_{c,} \min >T_{c, \mathrm{e}}\right)$, the rate $\mathrm{d} T_{\mathrm{c}} / \mathrm{d} t$ of temperature increase and the maximum test temperature $T_{\mathrm{c}, \max }$ As $T_{\mathrm{c}}$ 


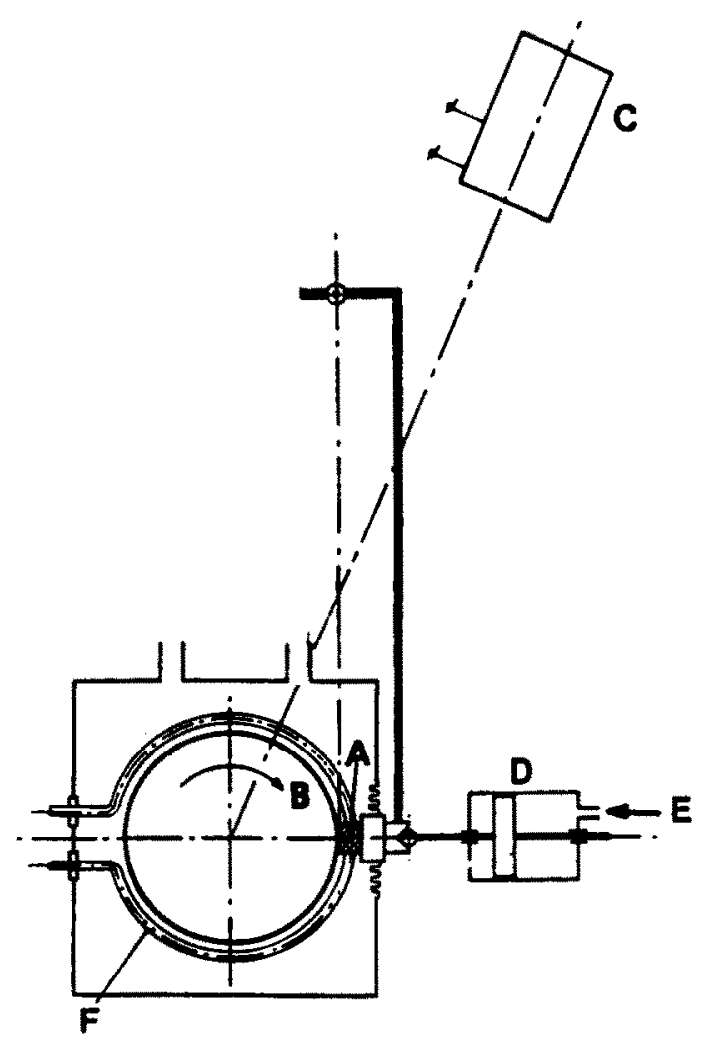

Fig. 1. Main components of the TNO tribometer: A, polymer block; $\mathrm{B}$, steel ring; $\mathrm{C}$, torque meter; $\mathrm{D}$, cylinder with air pressurized piston; $E$, pressurized air; $F$, inductive heating.

increases linearly with time, $f$ is also measured as a function of time $t$ or, at constant sliding speed $v$, as a function of sliding distance $d$.

The present tests were performed under the following conditions: steel ring surface roughness $R_{\mathrm{q}}=0.1 \mu \mathrm{m}$ (r.m.s. value), $F_{\mathrm{N}}=150 \mathrm{~N}, p=1.5 \mathrm{MPa}, v=0.1 \mathrm{~m} \mathrm{~s}^{-1}, T_{c, \min }=50$ ${ }^{\circ} \mathrm{C}, \mathrm{d} T_{\mathrm{c}} / \mathrm{d} t=15{ }^{\circ} \mathrm{C} \mathrm{h}^{-1}$ and $T_{\mathrm{c}, \max }=300{ }^{\circ} \mathrm{C}$. The experiments were performed in an environment of air of temperature $20-22{ }^{\circ} \mathrm{C}$ and relative humidity between $40 \%$ and $60 \%$.

Before measurement of an $f-T_{\mathrm{c}}$ curve, the specimens were run in for $20 \mathrm{~h}$ under a normal force $F_{\mathrm{N}}$ of $150 \mathrm{~N}$ and at a sliding speed $v=0.1 \mathrm{~m} \mathrm{~s}^{-1}$. During this runningin process the contact surface of the polymer block fully conformed to the curved surface of the steel ring as a result of mild wear. Thus the size of the contact surface and, with that, the contact pressure $p$ remained constant during the measurement of an $f-T_{c}$ curve.

\subsection{Performance of $20 \mathrm{~h}$ tests}

The $20 \mathrm{~h}$ tests were performed under the following conditions: steel ring surface roughness $R_{\mathrm{q}}=0.1 \mu \mathrm{m}$ (r.m.s. value), $F_{\mathrm{N}}=500 \mathrm{~N}, p=5 \mathrm{MPa}, v=0.25 \mathrm{~m} \mathrm{~s}^{-1}$. Once more the experiments were performed in an environment of air of temperature 20-22 ${ }^{\circ} \mathrm{C}$ and relative humidity between $40 \%$ and $60 \%$ or in water of temperature $20{ }^{\circ} \mathrm{C}$. 


\section{Results}

Before the results are presented and discussed, it should be noted that the mechanical properties and, in particular, the tribological properties of polymers may depend quite strongly on the production history of the materials as well as on the roughness of the steel mating surface. This is, for instance, shown by Lancaster [1].

Thus application of the results in practice needs careful consideration. Generally application-directed testing, in which the relevant practical conditions are simulated in the laboratory, will be necessary if a particular material is to be chosen for a given application; see work by de Gee [10].

\section{1. $f-T_{\mathrm{c}}$ curves}

Figure 2 shows the $f-T_{\mathrm{c}}$ curves for the 18 different combinations of polymers and fillers that were studied.

With the exception of the curves produced with POM, Fig. 2 presents a consistent picture: (1) high to very high friction for the unfilled materials over the full temperature range (as determined either by melting of the polymer or the attainment of $T_{\mathrm{c} \text {, max, }}$ in this case $300^{\circ} \mathrm{C}$ ); (2) a drastic reduction in $f$ over an appreciable part of the temperature range, when using PTFE-filled materials; (3) re-establishment of high $f$ values as a result of adding glass fibre to the materials, although the results still look more favourable than those produced with the unfilled materials.

Before the results, shown in Fig. 2, are analysed in more detail, it should be stated that in the case of $f-T_{\mathfrak{c}}$ curves for bearing materials meant for application under dry sliding conditions, two aspects are of importance, i.e. firstly the magnitude of the coefficient of friction $f$ and secondly the temperature $T^{*}$, up to which the polymer exhibits a "technically acceptable" behaviour as to friction. (A precise definition of the qualification "technically acceptable" cannot be given. Depending on the application one may select a criterion relating to the dissipation of frictional energy (i.e. to $f$ itself) or to the amount of wear caused by the tangential forces which result from friction.)

When these criteria are applied, Fig. 2 conveys the general message that from the polymers which were tested, only the PTFE-filled materials stand a good chance of functioning adequately as dry running bearing materials. Possibly, only POM forms an exception to this, because the unfilled version of this polymer exhibits a relatively low $f$ value over the entire measuring range. Addition of PTFE to this material results in a further reduction in $f$ only at temperatures over $70^{\circ} \mathrm{C}$. However, POM melts at $T=150^{\circ} \mathrm{C}$, which undoubtedly limits its applicability at higher environmental temperatures or at higher rates of frictional energy production.

Values of the maximum allowable temperature $T^{*}$, determined either by a sudden increase in friction or by melting, can be found easily for the materials with PTFE, but without glass fibre. These $T^{*}$ values are listed in Table 1 . It can be seen that in particular PEEK + PTFE, PPS + PTFE and PEI + PTFE are suited for use at relatively high contact temperatures.

\section{2. $20 \mathrm{~h}$ tests in air}

The individual results of the $20 \mathrm{~h}$ tests in air are presented in Table 2 . In this table the " $k$ values" are the average values of the specific wear rate $k$, measured after $20 \mathrm{~h}$ of testing. These values were calculated from data on the weight loss as a result of wear. The " $f_{\mathrm{e}}$ values" are the equilibrium values of the coefficient of friction $f$, 


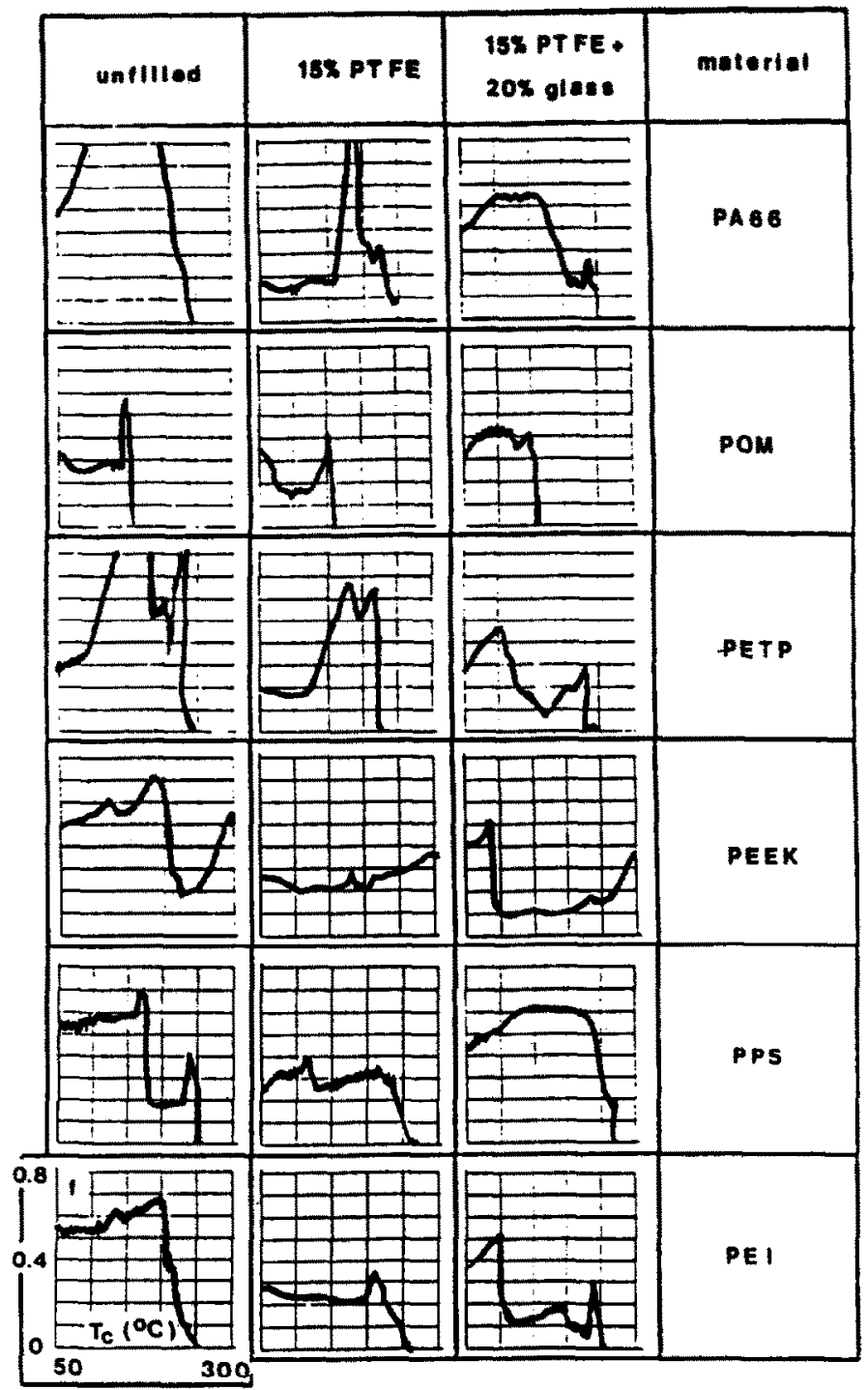

Fig. 2. Coefficient of friction $f v s$. contact temperature $T$ curves for 18 polymers: $F_{\mathrm{N}}=150 \mathrm{~N}$, $p=1.5 \mathrm{~N} \mathrm{~mm}^{-2}, v=0.1 \mathrm{~m} \mathrm{~s}^{-1}, \mathrm{~d} T_{\mathrm{c}} / \mathrm{d} t=15^{\circ} \mathrm{C} \mathrm{h}^{-1}$.

measured under conditions of thermal equilibrium, which was always reached within $0.5 \mathrm{~h}$.

From Table 2 it can be seen that the reproducibility of the test results is generally very good. Because of this the subsequent analysis always concerns the $k$ and $f_{\mathrm{e}}$ values averaged over the two duplicate tests.

Figure 3 shows the average values of the decrease in height $\Delta h / t\left(\mu \mathrm{m} \mathrm{h}^{-1}\right)$ per hour for the polymer blocks. Seen from left to right, the results per material are given in the order unfilled, filled with PTFE and filled with PTFE and glass fibre. The relevant $\Delta h / t$ values were calculated from the average $k$ values from Table 2 . The 
TABLE 1

$T^{*}$ values for the polytetrafluoroethylene-containing materials tested in air

\begin{tabular}{ll}
\hline Material & $T^{*}\left({ }^{\circ} \mathrm{C}\right)$ \\
\hline PA 66-PTFE & 150 \\
POM-PTFE & 150 \\
PETP-PTFE & 130 \\
PEEK-PTFE & 200 \\
PPS-PTFE & 220 \\
PEI-PTFE & 200 \\
\hline
\end{tabular}

values above the bars denote the values of $f_{c}$ multiplied by 100 . As the value of the equilibrium contact temperature $T_{c, e}$ depends on the amount of frictional energy that is released per unit of time, $T_{c, c}$ varies with $f_{s}$. The lowest $T_{c, e}$ value $\left(58^{\circ} \mathrm{C}\right)$ was measured during one of the experiments with PA 66-PTFE $\left(f_{\mathrm{e}}=0.13\right)$; the highest value $\left(197^{\circ} \mathrm{C}\right)$ was measured during one of the tests with PETP $\left(f_{\mathrm{e}}=0.71\right)$.

The wear results given in Table 2 and Fig. 3 confirm the previous conclusion that, with the exception of POM, the unfilled materials are not suited for use under conditions of dry running against steel in air. In this respect it should be noted that a $k$ value of $1 \times 10^{-15} \mathrm{~m}^{3} \mathrm{~N}^{-1} \mathrm{~m}^{-1}$ should be considered to be already on the high side for most practical applications; see Verbeek [11], de Gee et al. [12] and Thijsse [13]. The PTFE-filled polymers as well as those filled with PTFE + glass fibre exhibit a much better wear behaviour, whereas the materials without glass fibre perform better than those with glass fibre. PA 66-PTFE, POM-PTFE, PETP-PTFE and PEEK-PTFE perform exceptionally well, with $k$ values appreciably below $1 \times 10^{-15} \mathrm{~m}^{3} \mathrm{~N}^{-1} \mathrm{~m}^{-1}$.

The $f_{e}$ values confirm the expectation that a low wear rate occurs in conjunction with a low coefficient of friction: the materials with $k$ values lower than $1 \times 10^{-15} \mathrm{~m}^{3}$ $\mathrm{N}^{-1} \mathrm{~m}^{-1}$ have $f_{\mathrm{e}}$ values of 0.21 or lower.

As indicated in Table 2, with materials based on PPS a thick layer of transferred polymer invariably forms on the steel ring surface. Similar severe transfer phenomena are not found with the other materials (note that transferred PTFE films that may form when PTFE-containing polymers are rubbed against steel are not visible with the naked eye).

Finally, it is found that the use of PEEK-PTFE-glass or PEI-PTFE-glass leads to the formation of a few shallow (approximately $5 \mu \mathrm{m}$ deep) scratches on the steel ring surface ("light scratching").

\subsection{0 h tests in water}

The results of the experiments performed in water are presented in Table 3 and Fig. 4.

If we look firstly at the $f_{\mathrm{e}}$ values, it can be seen that testing in water instead of in air generally leads to a considerable reduction in friction. However, with one exception (PETP-PTFE-glass fibre; see below), the relevant $f_{e}$ values are still of the order of 0.1 or higher, i.e. characteristic for boundary lubrication rather than hydrodynamic lubrication.

Comparison with the results found in dry air (Table 2 and Fig. 3) shows that testing in dry air certainly does not yield useful indications regarding the behaviour of the materials in water. This once more shows that statements on the wear resistance 
TABLE 2

Numerical results of the $20 \mathrm{~h}$ tests in air

\begin{tabular}{|c|c|c|c|}
\hline Material & $\begin{array}{l}k \\
\left(\times 10^{-15} \mathrm{~m}^{3} \mathrm{~N}^{-1} \mathrm{~m}^{-1}\right)\end{array}$ & $f_{\mathrm{e}}$ & Ring $^{a}$ \\
\hline \multirow[t]{2}{*}{ PA 66} & 18.8 & 0.58 & + \\
\hline & 13.0 & 0.56 & + \\
\hline \multirow[t]{2}{*}{ PA 66-PTFE } & 0.5 & 0.13 & + \\
\hline & 0.6 & 0.14 & + \\
\hline \multirow[t]{2}{*}{ PA 66-PTFE-glass } & 2.1 & 0.29 & + \\
\hline & 1.8 & 0.34 & + \\
\hline \multirow[t]{2}{*}{ POM } & 2.0 & 0.44 & + \\
\hline & 2.2 & 0.46 & + \\
\hline \multirow[t]{2}{*}{ POM-PIFE } & 0.4 & 0.22 & + \\
\hline & 0.4 & 0.21 & + \\
\hline \multirow[t]{2}{*}{ POM-PTFE-glass } & 4.3 & 0.25 & + \\
\hline & 3.9 & 0.21 & + \\
\hline \multirow[t]{2}{*}{ PETP } & 21.8 & 0.71 & + \\
\hline & 21.6 & 0.66 & + \\
\hline \multirow[t]{2}{*}{ PETP-PTFE } & 0.6 & 0.16 & + \\
\hline & 0.8 & 0.13 & + \\
\hline \multirow[t]{2}{*}{ PETP-PTFE-glass } & 1.9 & 0.19 & + \\
\hline & 1.7 & 0.18 & + \\
\hline \multirow[t]{2}{*}{ PEEK } & 14.8 & 0.51 & + \\
\hline & 14.1 & 0.48 & + \\
\hline \multirow[t]{2}{*}{ PEEK-PTFE } & 0.6 & 0.19 & + \\
\hline & 0.5 & 0.17 & + \\
\hline \multirow[t]{2}{*}{ PEEK-PTFE-glass } & 1.3 & 0.21 & $+1-$ \\
\hline & 1.1 & 0.19 & $+1-$ \\
\hline \multirow[t]{2}{*}{ PPS } & 37.5 & 0.78 & Transfer \\
\hline & 39.7 & 0.63 & Transfer \\
\hline \multirow[t]{2}{*}{ PPS-PTFE } & 2.9 & 0.31 & Transfer \\
\hline & 2.8 & 0.29 & Transfer \\
\hline \multirow[t]{2}{*}{ PPS-PTFE-glass } & 3.6 & 0.42 & Transfer \\
\hline & 3.3 & 0.36 & Transfer \\
\hline \multirow[t]{2}{*}{ PEI } & 46.6 & 0.45 & + \\
\hline & 29.6 & 0.41 & + \\
\hline \multirow[t]{2}{*}{ PEI-PTFE } & 2.4 & 0.22 & + \\
\hline & 2.4 & 0.21 & + \\
\hline \multirow[t]{2}{*}{ PEI-PTFE-glass } & 2.7 & 0.22 & $+1-$ \\
\hline & 3.3 & 0.20 & $+1-$ \\
\hline
\end{tabular}

Ring material, hardened steel (AISI 52100), $R_{\mathrm{q}}=0.1 \mu \mathrm{m} ; F_{\mathrm{N}}-500 \mathrm{~N} ; p=5 \mathrm{~N} \mathrm{~mm}^{-2} ; v=0.25$ $\mathrm{m} \mathrm{s}^{-1}$.

${ }^{a}+$, surface has not visibly changed by contact with the polymer; $+1-$, light scratching of the ring surface.

of materials are unrealistic as long as the environment in which the tests were performed is not carefully specified. Contrary to the situation in air, addition of PTFE to PA 66, PETP and PEEK does not lead to a decrease in the wear rate in water and even has a negative effect in the case of POM. With unfilled PPS and PEI, testing in water leads to a dramatic increase in the already high wear rates. In this respect the behaviour of PTFE-filled PPS is quite remarkable, as in this case PTFE still seems to fulfil its 


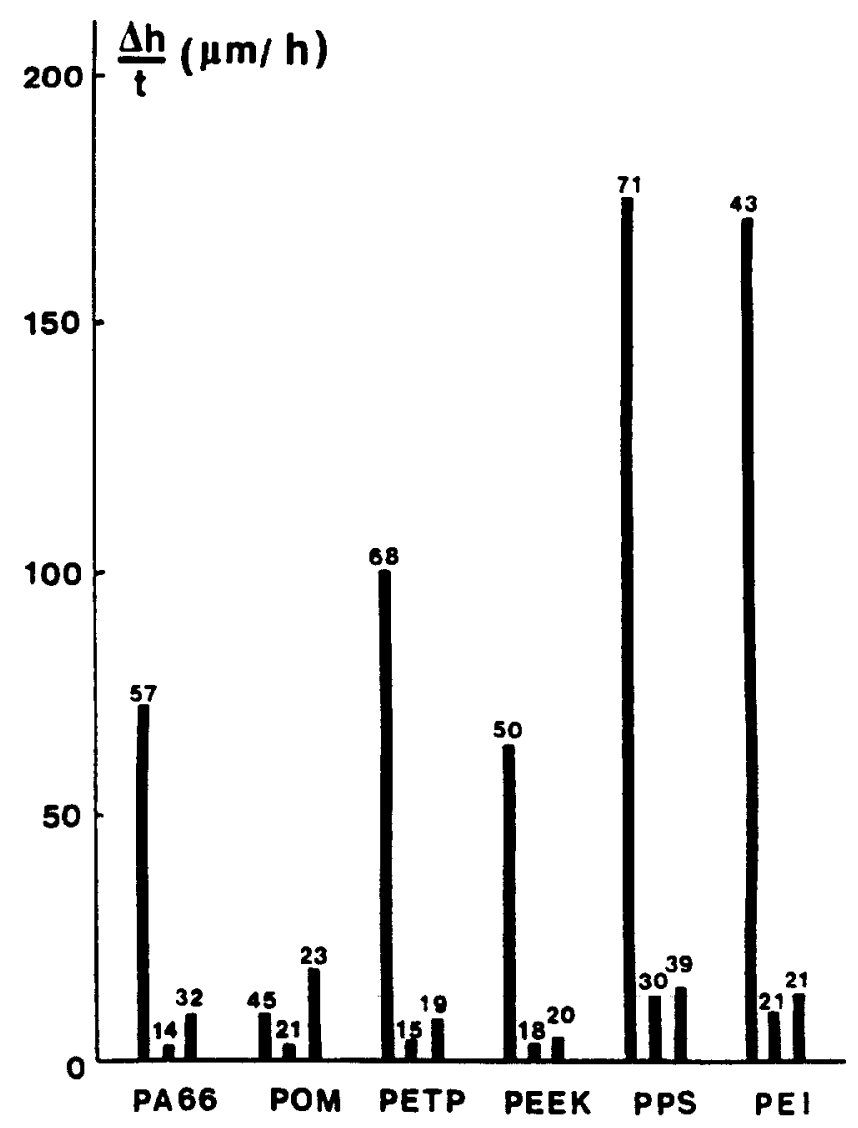

Fig. 3. Results of the $20 \mathrm{~h}$ tests performed in dry air. For test parameters see Table 2. The heights of the bars denote the average decreases in height $\Delta h / t\left(\mu \mathrm{m} \mathrm{h}^{-1}\right)$ of the polymer blocks per hour. For each polymer the following sequence was applied: "unfilled", "filled with PTFE" and "filled with PTFE and glass fibre." The values above the bars denote the average equilibrium values of the coefficients of friction $f_{e}$, multiplied by 100 . A $\Delta h / t$ value of $4.5 \mu \mathrm{m} \mathrm{h}^{-1}$ corresponds to an average $k$ value of $1.10^{15} \mathrm{~m}^{3} \mathrm{~N}^{-1} \mathrm{~m}^{-1}$.

wear-reducing function (albeit that with a $k$ value of approximately $4 \times 10^{-15} \mathrm{~m}^{3} \mathrm{~N}^{-1}$ $\mathrm{m}^{-1}$ PPS-PTFE is still a rather dubious choice).

Addition of glass fibre to PTFE-containing PA 66, PEEK and PPS is found to lead to a considerable increase in wear rate when the tests are performed in water. With PETP-PTFE-glass fibre an initially high $k$ value decreases to zero, which is accompanied by a reduction in $f_{e}$ to values below 0.01 . Such tow $f_{e}$ values are indicative of the fact that hydrodynamic separation of the surfaces of polymer and steel ring occurred after running for some $2 \mathrm{~h}$ at $v=0.25 \mathrm{~m} \mathrm{~s}^{-1}$. Inspection of the steel ring surfaces after termination of the tests reveals that this is due to a considerable reduction in surface roughness of the steel. Undoubtedly this is a result of a polishing action of the glass fibres. It is not clear why a similar polishing effect does not occur with the other glass-fibre-containing materials. Obviously with this material, as well as with the other materials, hydrodynamic lubrication conditions at $v=0.25 \mathrm{~m} \mathrm{~s}^{-1}$ may well be established from the very start of the tests if the mating steel surfaces are polished 
TABLE 3

Numerical results of the $20 \mathrm{~h}$ tests in water

\begin{tabular}{|c|c|c|c|}
\hline Material & $\begin{array}{l}k \\
\left(\times 10^{-15} \mathrm{~m}^{3} \mathrm{~N}^{-1} \mathrm{~m}^{-1}\right)\end{array}$ & $f_{e}$ & Ring ${ }^{a}$ \\
\hline \multirow[t]{2}{*}{ PA 66} & 1.1 & 0.14 & + \\
\hline & 0.7 & 0.09 & + \\
\hline \multirow[t]{2}{*}{ PA 66-PTFE } & 0.9 & 0.19 & + \\
\hline & 0.5 & 0.08 & + \\
\hline \multirow[t]{2}{*}{ PA 66-PTFE-glass } & 26.9 & 0.23 & $+1-$ \\
\hline & 31.8 & 0.10 & $+1-$ \\
\hline \multirow[t]{2}{*}{ POM } & 0.6 & 0.13 & + \\
\hline & 1.0 & 0.13 & + \\
\hline \multirow[t]{2}{*}{ POM-PTFE } & 2.3 & 0.15 & + \\
\hline & 1.6 & 0.20 & + \\
\hline \multirow[t]{2}{*}{ POM-PTFE-glass } & 3.2 & 0.20 & + \\
\hline & 3.4 & 0.20 & + \\
\hline \multirow[t]{2}{*}{ PETP } & 9.6 & 0.12 & + \\
\hline & 10.5 & 0.13 & + \\
\hline \multirow[t]{2}{*}{ PETP-PTFE } & 11.1 & 0.10 & + \\
\hline & 8.8 & 0.12 & + \\
\hline \multirow[t]{2}{*}{ PETP_PTFE-glass } & $18.6^{\mathrm{b}}$ & $<0.01^{\mathrm{b}}$ & $++^{c}$ \\
\hline & $10.1^{\mathrm{b}}$ & $<0.01^{\mathrm{b}}$ & $+c$ \\
\hline \multirow[t]{2}{*}{ PEEK } & 1.4 & 0.12 & + \\
\hline & 1.2 & 0.11 & + \\
\hline \multirow[t]{2}{*}{ PEEK-PTFE } & 1.6 & 0.10 & + \\
\hline & 1.0 & 0.08 & + \\
\hline \multirow[t]{2}{*}{ PEEK-PTFE-glass } & 31.7 & 0.26 & Transfer $^{d}$ \\
\hline & 31.4 & 0.24 & Transfer ${ }^{d}$ \\
\hline \multirow[t]{2}{*}{ PPS } & 50.0 & 0.26 & + \\
\hline & 51.3 & 0.24 & + \\
\hline \multirow[t]{2}{*}{ PPS-PTFE } & 3.7 & 0.10 & Transfer ${ }^{d}$ \\
\hline & 4.0 & 0.09 & Transfer ${ }^{d}$ \\
\hline \multirow[t]{2}{*}{ PPS-PTFE-glass } & 51.5 & 0.48 & + \\
\hline & 47.3 & 0.25 & + \\
\hline \multirow[t]{2}{*}{ PEI } & 144.0 & 0.25 & + \\
\hline & 139.0 & 0.24 & + \\
\hline \multirow[t]{2}{*}{ PEI-PTFE } & 29.5 & 0.16 & + \\
\hline & 25.6 & 0.15 & + \\
\hline \multirow[t]{2}{*}{ PEI-PTFE_glass } & 29.3 & 0.40 & + \\
\hline & 27.3 & 0.36 & + \\
\hline
\end{tabular}

Ring material, stainless steel (AISI 316), $R_{\mathrm{q}}=0.1 \mu \mathrm{m} ; F_{\mathrm{N}}-500 \mathrm{~N} ; p=5 \mathrm{~N} \mathrm{~mm}^{-2} ; v=0.25 \mathrm{~m}$ $\mathrm{s}^{-1}$.

+ , surface has not visibly changed by contact with the polymer; $+1-$, light scratching of the ring surface.

${ }^{\mathrm{b}}$ After some time the contacting surfaces of polymer and ring become completely separated by a thin water film (hydrodynamic lubrication).

${ }^{\mathrm{C}} \mathrm{Ring}$ acquires a mirror polish.

'Transferred layer is very thin. 


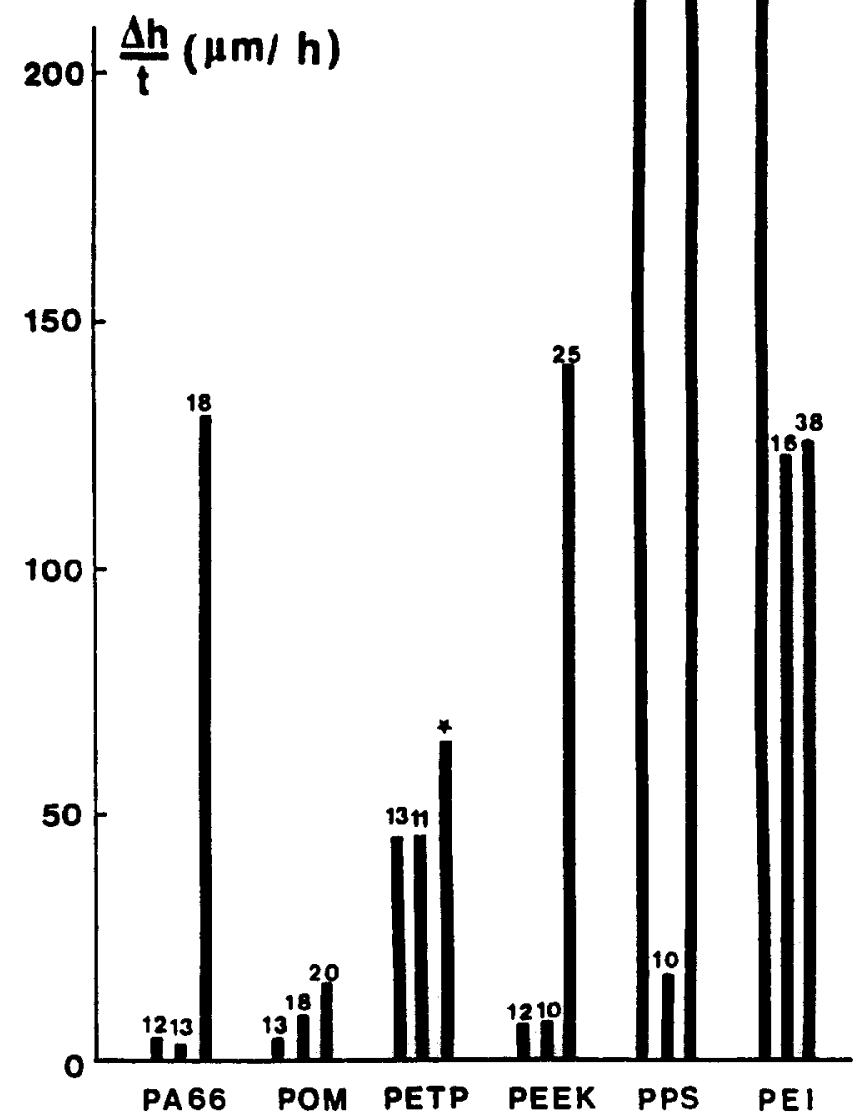

Fig. 4. Results of the $20 \mathrm{~h}$ tests performed in water. For test parameters see Table 4. For explanation see the caption of Fig. 3.

before the test. In practice, this may be a complete, but expensive, solution of the wear problem.

If the $k<1 \times 10^{-15} \mathrm{~m}^{3} \mathrm{~N}^{-1} \mathrm{~m}^{-1}$ criterion is applied, it can be concluded that PA 66, PA 66-PTFE and POM qualify as materials particularly suitable for application in water. Further, if a high initial (running-in) wear rate can be tolerated, PETP-PTFE-glass also may perform adequately.

\subsection{The effect of variations in polytetrafluoroethylene content on the behaviour of} polyamide 66

Above it was shown that PTFE plays a crucial role in the establishment of low friction and wear values if the experiments are performed in air. Thus it was thought expedient to study the effect of variations in PTFE content on the behaviour of at 
least one of the polymers. For this PA 66 was selected. Figure 5 shows $f-T_{\mathrm{c}}$ curves obtained with PA 66 with $0 \%, 5 \%, 10 \%, 15 \%$ and $20 \%$ PTFE.

It can be seen that a decrease in PTFE content from the "usual" $15 \%$ to $10 \%$ hardly affects the $f-T_{\mathrm{c}}$ curve. The only effect is a small increase in $f$ for $T_{\mathrm{c}}<T^{*}$.

Increasing the PTFE percentage to $20 \%$ does not affect $f$ for $T_{c}<T^{*}$, while in the region $T_{c}>T^{*}$ a drastic decrease in $f$ is found. The latter effect probably has no implications for practical use.

The results of $20 \mathrm{~h}$ tests in air performed with these materials can be found in Table 4. It turns out that when PA 66 is used with 15\% PTFE the lowest values of $f$ and $k$ are measured.

To summarize these results, it can be concluded that for PA 66 a PTFE percentage of $15 \%$ is a good choice.

\section{Discussion}

The beneficial effect of PTFE in air can be explained by assuming that a thin transferred, friction-reducing film of PTFE forms on the mating steel surface. Such

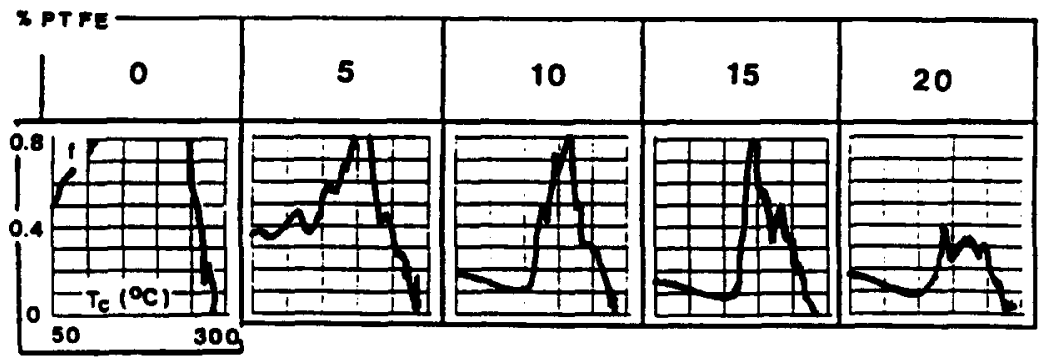

Fig. 5. $f-T_{\mathrm{c}}$ curves for PA 66 with $0 \%, 5 \%, 10 \%, 15 \%$ and $20 \%$ PTFE: $F_{\mathrm{N}}=150 \mathrm{~N}, p=1.5 \mathrm{~N}$ $\mathrm{mm}^{-2}, v=0.1 \mathrm{~m} \mathrm{~s}^{-1}, \mathrm{~d} T_{\mathrm{c}} / \mathrm{d} t=15^{\circ} \mathrm{C} \mathrm{h}^{-1}$.

\section{TABLE 4}

Numerical results of the $20 \mathrm{~h}$ tests in air with polyamide 66 with different percentages of polytetrafluoroethylene

\begin{tabular}{lll}
\hline $\begin{array}{l}\text { Percentage } \\
\text { PIFE }\end{array}$ & $k$ & $f_{\mathrm{e}}$ \\
\hline 0 & 14.0 & 0.58 \\
& 12.4 & 0.60 \\
5 & 2.2 & 0.28 \\
& 3.6 & 0.33 \\
10 & 5.1 & 0.19 \\
& 4.3 & 0.18 \\
15 & 1.8 & 0.15 \\
& 2.4 & 0.16 \\
20 & 4.0 & 0.18 \\
& 2.8 & 0.17
\end{tabular}

Ring material, hardened steel (AISI 52100 ), $R_{\mathrm{q}}-0.1 \mu \mathrm{m} ; F_{\mathrm{N}}-500 \mathrm{~N} ; p=5 \mathrm{~N} \mathrm{~mm}^{-2} ; v-0.25$ $\mathrm{m} \mathrm{s}^{-1}$. 
films have been found by Wheeler [14], Lauer et al. [15] and Odi-Owei and Schipper [16]. Water probably inhibits the formation of such a transferred film.

In discussing the wear results in more detail it is quite helpful to introduce firstly the "rule of thumb" that wear increases with the ratio of $f$ over $S$, in which $S$ is the "strength" of the surface zone of the polymer under conditions of combined normal and tangential loading (in quantitative treatments $S$ will stand for fracture strength, fatigue strength or shear strength, depending on the type of wear mechanism that predominates). This rule implies that a decrease in $f$ as well as an increase in $S$ may result in improved wear characteristics. In developing new materials a combined action is even more attractive, i.e. decrease in $f$ and increase in $S$.

On considering the above, it is of interest to subject the results obtained with the glass-fibre-containing materials to a further analysis. It is found that the relevant materials can be classified in five groups, as shown in Table 5.

In the case of the base materials in groups 1,2 and 3 friction seems to be the only wear-controlling factor. For PEEK and PEI a small increase in $f$ is accompanied by a small increase in wear rate, for PA $66 f_{e}$ as well as the wear rate increase by a factor of 2, while for PETP both increases are medium. This behaviour is characteristic for materials which do not suffer from an appreciable change in $S$ on addition of glass fibre.

For the base material in group 4 (POM) addition of glass fibre results in a considerable increase in wear rate, although the effect on $f_{e}$ is small. Probably for this material glass fibre deleteriously affects $S$.

For PPS, finally, this effect seems to be reversed.

Further, it is found that testing in water instead of in air leads to dramatic increases in the wear rate of unfilled PPS and PEI, while addition of glass fibre to PTFE-containing PA 66, PEEK and PPS has the same effect, if the tests are performed in water. These effects may well be caused by penetration of water into the surface zones of the polymers, causing a corresponding decrease in $S$. A similar effect has been found to be responsible for the early failure of ball-bearings in water-containing lubricants; see work by Schatzberg and Felsen [17].

Actually the above hypotheses should be substantiated by measurement of the (changes in) surface strength of the different polymers in their corresponding environments. As yet such work has not yet been performed.

TABLE 5

The effect of adding glass fibre to polytetrafluoroethylene-containing polymers

\begin{tabular}{llll}
\hline Group & Material(s) & \multicolumn{2}{l}{ Relative increases in the following } \\
\cline { 4 - 4 } & & Friction & Wear \\
\hline 1 & PEEK, PEI & Small & Small \\
2 & PA 66 & Large & Large \\
3 & PETP & Medium & Medium \\
4 & POM & Small & Large \\
5 & PPS & Medium & Small \\
\hline
\end{tabular}




\section{Conclusions}

(1) $f-T$ curves, measured in air at a controlled rate of increase in contact temperature $T_{\mathrm{c}}$ and $20 \mathrm{~h}$ tests in air and in water at freely varying $T_{\mathrm{c}}$, together adequately describe the tribological behaviour of polymers in sliding contact with steel.

(2) In dry air the materials PA 66-PTFE, POM-PTFE, PETP-PTFE and PEEK-PTFE have $k$ values well below $1.0 \times 10^{-15} \mathrm{~m}^{3} \mathrm{~N}^{-1} \mathrm{~m}^{-1}$ and coefficients of friction $f$ of the order of $0.15-0.20$. Thus they seem excellently suited for application under conditions of dry running contact with steel. From these materials PEEK-PTFE has the additional advantage that it can sustain a relatively high temperature $\left(T^{*}=200\right.$ $\left.{ }^{\circ} \mathrm{C}\right)$.

(3) In water the materials PA 66, PA 66-PTFE and POM have $k$ values of $1.0 \times 10^{-15} \mathrm{~m}^{3} \mathrm{~N}^{-1} \mathrm{~m}^{-1}$ or lower at $f$ values of well below 0.20 . Thus these materials should form the first choice for application in water, under conditions where hydrodynamic film formation is not expected to occur. PETP-PTFE-glass may also be a good choice, because with this material polishing of the mating steel surface occurs to such an extent that hydrodynamic lubrication conditions are casily established.

(4) From the above it can be concluded that in dry air the presence of PTFE in the polymers is essential if good tribological properties in sliding contact against steel are to be obtained. In an environment of water this does not seem to be the case, probably because of the fact that in water a transferred film of PTFE does not form.

Regarding the above conclusions it should be noted that materials which in the present experiments performed less satisfactorily may nevertheless be applicable quite well under certain practical conditions.

Also, variations in "production history" of the polymers, in the surface roughness of the mating steel surface or in the test conditions may affect the quality ranking that is obtained.

Still, in the absence of contradictory evidence, the present authors consider it fair to assume that the crucial effect of PTFE, which has been found in dry air, is of general significance for practice.

\section{Acknowledgments}

The authors gratefully acknowledge financial support received from the Dutch Ministries of Economic Affairs and Transportation and Public Works and the industrial companies DAF Trucks, DSM Research, ENKA Research, Fokker, Grasso-Stacon, IHC-MTI Holland, Jonge Poerink, Philips CFT, Stork Pompen and Thomassen International.

\section{References}

1 J. K. Lancaster, Polymer based bearing materials, the role of fillers and fibre reinforcement, Tribol. Int., 5 (1972) 249-255.

2 J. K. Lancaster, Dry bearings: a survey of materials and factors affecting their performance, Tribol. Int., 6 (1973) 219-252.

$3 \mathrm{~K}$. Tanaka, Friction and wear of glass and carbon fibre filled thermoplastic polymers, $J$. Lubr. Technol., 99 (1977) 408-414. 
4 B. J. Briscoe, Wear of polymers: an essay on fundamental aspects, Tribol. Int., 14 (1981) 231-243.

5 D. C. Evans and G. S. Senior, Self lubricating materials for plain bearings, Tribol. Int., 15 (1982) 243-248.

6 E. Santner and H. Czichos, Tribology of polymers, Tribol. Int. 22 (1989) 103-109.

7 R. L. Fusaro, Self lubricating polymer compusites and polymer transfer film lubrication for space applications, Tribol. Int., 23 (1990) 105-122.

8 A. C. M. Honselaar and A. W. J. de Gee, Dynamic thermo-analytical test for qualifying brake lining materials, Tribol. Int., 18 (1985) 21-27.

9 A. C. M. Honselaar and A. W. J. de Gee, Dynamic loadibility of polymer-metal friction couples, in K. Holmberg (ed.), Proc. 5th Int. Congr. on Tribology "EUROTRIB", Helsinki, 1989, pp. 282-288.

10 A. W. J. de Gee, Selection of materials for tribotechnical applications, Tribol. Int., 11 (1979) 233-365.

11 H. J. Verbeek, Tribological systems and wear factors, Wear, 56 (1979) 81-92.

12 A. W. J. de Gee, A. Begelinger and G. Salomon, Lubricated wear of steel point contacts - application of the transition diagram. In K. C. Ludema (eds.), Proc. Int. Conf. on Wear of Materials, Reston, VA, 1983, American Society of Mechanical Engineers, New York, 1983, pp. 534-540.

13 C. J. Thijsse, Tribology aids for designers. In D. Dowson, C. M. Taylor, M. Godet and D. Berthe (eds.), Tribological Design for Machine Elements, Elsevier, Amsterdam, 1989, pp. $495-499$.

14 D. A. Wheeler, The transfer of PTFE studied by X-ray photoelectron spectroscopy, Wear, 66 (1980) 355-365.

15 J. L. Lauer, B. G. Bunting and W. R. Jones, Jr., Investigation of PTFE transfer films by infrared emission spectroscopy and phase-locked ellipsometry, STLE Tribol. Trans., 31 (1988) 282-288.

16 S. Odi-Owei and D. J. Schipper, Tribological behaviour of unfilled and composite POM, Wear, to be published.

17 P. Schatzberg and I. M. Felsen, Effects of water and oxygen during rolling contact lubrication, Wear, 12 (1968) 331-342.

\section{Appendix A: Nomenclature}

$f \quad$ coefficient of friction

$F_{\mathrm{N}} \quad$ normal force $(\mathrm{N})$

$k \quad$ specific wear rate $\left(\mathrm{m}^{3} \mathrm{~N}^{-1} \mathrm{~m}^{-1}\right)$

$p \quad$ apparent contact pressure $\mathrm{MPa}$

$R_{\mathrm{q}} \quad$ standard deviation in the distribution of roughness heights (r.m.s. value) $(\mu \mathrm{m})$

$S$ strength of surface zone under combined normal and tangential loading (MPa)

$T$ environmental temperature $\left({ }^{\circ} \mathrm{C}\right)$

$T_{\mathrm{c}} \quad$ contact temperature $\left({ }^{\circ} \mathrm{C}\right)$

$v \quad$ sliding velocity $\left(\mathrm{m} \mathrm{s}^{-1}\right)$

\section{Subscripts and superscripts}

e equilibrium value

* applicability limit 